\title{
Induction of apoptosis and inhibition of cell adhesive and invasive effects by tanshinone IIA in acute promyelocytic leukemia cells in vitro
}

\author{
Jia-Jun Liu ${ }^{1, *, \dagger}$, Dong-Jun Lin ${ }^{1, \dagger}$, Pei-Qing Liu ${ }^{2, *}$, Min Huang ${ }^{2}$, Xu-Dong Li $^{1}$ \& \\ Ren-Wei Huang ${ }^{1}$ \\ ${ }^{1}$ Department of Hematology and Oncology, The Third Affiliated Hospital of Sun Yat-sen University, \\ Guangzhou, 510630, P.R. China; ${ }^{2}$ Department of Pharmacology and Toxicology, School of Pharmaceutical \\ Sciences, Sun Yat-Sen University, Guangzhou, 510080, P.R. China
}

Received 27 May 2006; accepted 2 August 2006

(C) 2006 National Science Council, Taipei

Key words: apoptosis, Bcl-2, caspase-3, extracellular matrix (ECM), leukemia, mitochondrial membrane potential, tanshinone IIA

\begin{abstract}
Tanshinone IIA, a diterpene quinone extracted from the traditional herbal medicine, Salvia miltiorrhiza Bunge, is used widely and successfully in clinics in China for treating inflammatory diseases. Recently tanshinone IIA has been reported to have apoptosis inducing effects on a large variety of cancer cells. In this study, the anti-proliferation and apoptosis inducing effects of tanshinone IIA as well as its influence on cell adhesion to and invasion through the extracellular matrix (ECM) on acute promyelocytic leukemia (APL) NB4 cells in vitro were studied. Cell proliferation was assessed by MTT assay, cell apoptosis was observed by Hoechst 33258 staining and flow cytometry (FCM); The variation of caspase- 3 and apoptotic related genes were assayed by Western blotting, cell mitochondrial membrane potential as well as cell adhesive and invasive effects were also investigated by using standard methods. The results showed that tanshinone IIA exhibited induction of apoptosis by activation of caspase-3, downregulation of antiapoptotic protein bcl-2 and bcl-xl and upregulation of pro-apoptotic protein bax, as well as disruption of the mitochondrial membrane potential. Furthermore, treatment by tanshinone IIA could reduce cell adhesion to and invasion through ECM in leukemia NB4 cells. These data provide a potential mechanism for tanshinone IIA-induced apoptosis and cell growth inhibition in leukemia NB4 cells, suggesting that tanshinone IIA may serve as an effective adjunctive reagent for the treatment of APL.
\end{abstract}

\section{Introduction}

Acute myeloid leukemia (AML) is a group of several different diseases, the treatment and outcome of which depend on several factors, including leukemia karyotype, patient age, and comorbid conditions. Despite advances in understanding the molecular biology of AML, its treatment remains

*To whom correspondence should be addressed. E-mail: jiajun.1@163.com; sps02@gzsums.edu.cn

${ }^{\dagger}$ Contributed equally to this study. challenging. Standard regimens using cytarabine and anthracyclines for induction followed by some form of postremission therapy produce response rates of $60-70 \%$, with less than $20 \%$ of all patients achieving long-term disease-free survival [1, 2]. Acute promyelocytic leukemia (APL) is a rare disease accounting for approximately $10 \%$ of AML. This kind of leukemia is associated with a high risk of early mortality before the onset of therapy or in the early treatment phase resulting from severe coagulopathy, frequently inducing fatal cerebral hemorrhage, and the early mortality 
rate is approximately $10 \%$ [3]. Therefore, it is a permanent subject to find new anti-leukemia drugs and effective therapies for the clinical treatment of myeloid leukemia.

Recently, the use of herbal medicines to prevent the development as well as recurrence of a large variety of malignant diseases has become widely accepted as a realistic option for the treatment of malignant disease. There have been intense activities not only to identify new herbal medicines but also to understand how the existing constituents exhibit their activities [4]. Danshen (Salvia miltiorrhiza Bunge) is a widely used Chinese herbal medicine; its extracts contain diterpene quinone and phenolic acid derivatives, including tanshinone (I, IIA and IIB), cryptotanshinone, isocryptotanshinone, miltirone, tanshinol (I and II) and salviol [5]. Tanshinone IIA is a derivative of phenanthrene-quinone isolated from Danshen (Figure 1); it has anti-oxidant properties, inhibiting the association of lipid peroxidation products with DNA by breaking the chain reactions of peroxidation by scavenging lipid free radicals [6-8]. Recent studies have shown that Tanshinone IIA has significant anti-proliferation effects by inducing apoptosis against multiple human cancer cell lines such as human breast cancer [9] and hepatocellular carcinoma [10].

Though tanshinone IIA has been proved to have anti-tumor effects in many different human cancer cells, many of its anti-proliferation and apoptotic mechanisms remain to be demonstrated. To date, no detailed data are available about the role and mechanisms of tanshinone IIA in leukemia NB4 cells. In order to understand the roles of tanshinone IIA in NB4 cells and possible clinical application of tanshinone IIA in leukemia therapy, we examined the anti-proliferation effects of tanshinone IIA as well as its influence on cell adhesion

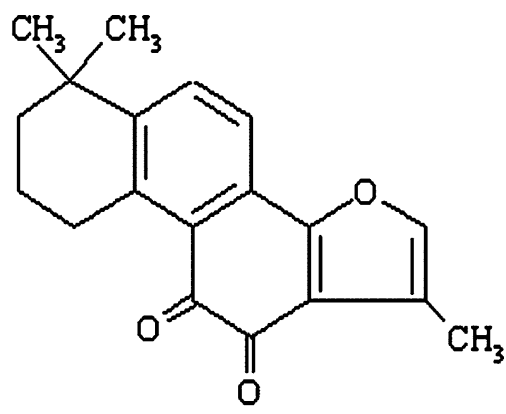

Figure 1. Molecular structure of tanshinone IIA. to and invasion through extracellular matrix (ECM) on NB4 cells in vitro.

\section{Materials and methods}

\section{Main reagents}

Tanshinone IIA, isolated from Salvia miltiorrhiza Bunge, was provided by Professor Gu Lian-Quan, Institute of Pharmacy Synthesis, Sun Yat-sen University. Annexin V/FITC and propidium iodide (PI) apoptosis detection kit was obtained from $R \& D$, USA. Hoechst 33258 was purchased from Sigma Company. The antibodies used in this study, antibcl-2, bcl-xl, bax, bid, bak and bad were purchased from Santa Cruz Company (Germany). Antibodies against caspase-3 was bought from Upstate Inc. Caspase inhibitor (z-DEVD-FMK) was purchased from R\&D systems Inc. (Minneapolis, MN, US). TRIZOL was from GIBCO (USA), and caspase colorimetric assay kit was from MBI (USA).

\section{Cell culture}

Leukemia NB4 cells were provided by central laboratory of Sun Yat-sen university cancer center. Cells were cultured in RPMI-1640 medium supplemented with $10 \%$ fetal calf serum (FBS), $100 \mathrm{U} / \mathrm{ml}$ penicillin and $100 \mu \mathrm{g} / \mathrm{ml}$ streptomycin, in a humidified incubator with $5 \% \mathrm{CO}_{2}$ at $37^{\circ} \mathrm{C}$. All the cells were passaged twice weekly and routinely examined for mycoplasma contamination. Cells in logarithmic growth phase were used for further experiments.

\section{Cell growth inhibition assay}

The cell growth inhibition effects caused by tanshinone IIA was assessed by MTT assay, which is based on the reduction of MTT by the mitochondrial dehydrogenase of intact cells to a purple formazan product. Briefly, leukemia NB4 cells in logarithmic growth-phase were collected, and $2 \times 10^{5}$ cells/well were dispensed within 96-well culture plates in $100 \mathrm{ml}$ volumes. Then different concentrations of tanshinone IIA $(10,20,30,40$ and $50 \mu \mathrm{mol} / 1)$ were put in different wells. Every one of the concentrations above was regarded as one treated group while there was no tanshinone IIA in the control group. Each of the treated or 
control group contained 6 parallel wells. Before MTT assay, the cells were first incubated in serum free RPMI for $24 \mathrm{~h}$. After tanshinone IIA was absorbed completely, culture plates were then maintained in RPMI containing $10 \%$ fetal calf serum for $0,12,24$ and $48 \mathrm{~h}$ prior to the addition of tetrazolium reagent. MTT working solution was prepared as follows: $5 \mathrm{mg} \mathrm{MTT} / \mathrm{ml} \mathrm{PBS}$ was sterile by being filtered with $0.45 \mu \mathrm{m}$ filter units. Each of the above cultured wells was added $20 \mu$ of MTT working solution and then incubated continuously for $4 \mathrm{~h}$. All culture medium supernatant was removed from each wells after centrifugation and replaced with $100 \mu \mathrm{l}$ of DMSO. Following thorough solubilization, the absorbance (OD) of each well was measured using a microculture plate reader at $570 \mathrm{~nm}$. Each condition was tested in triplicate. The growth inhibitory rate was calculated by the following formula:
Then hoechst 33258 staining was used to observe the apoptotic morphology. Cells were fixed with $4 \%$ formaldehyde in phosphate buffered saline (PBS) for $10 \mathrm{~min}$, stained by Hoechst 33258 $(10 \mathrm{mg} / \mathrm{l})$ for $1 \mathrm{~h}$, and then subjected to fluorescence microscopy. After treatment with tanshinone IIA, the morphologic changes including reduction in the volume and nuclear chromatin condensation were observed.

\section{Adhesion assay}

In vitro adhesion assays were performed to evaluate the effects of tanshinone IIA on the adhesive properties of NB4 cells. The plates for the adhesion assays were precoated with the ECM proteins laminin, fibronectin, vitronectin, or type IV collagen (each at a final concentration of $1 \mathrm{mg} / \mathrm{ml}$ in PBS) overnight at $4{ }^{\circ} \mathrm{C}$ and dried. To study the

$$
\begin{aligned}
\text { Growth inhibitory rate } & =(\text { average OD value in the control group } \\
& - \text { average OD value in the treatment group }) / \text { average OD value in the } \\
& \text { control group } \times 100 \% .
\end{aligned}
$$

The $50 \%$ inhibitory concentration (IC50) was determined from dose-response curve from at least three independent experiments. The percentages of cell growth were used to obtain the full doseresponse curves and to determine the IC50 values (concentration inhibiting of $50 \%$ the cell growth compared with control).

\section{Apoptosis assays}

After the cells treated with $40 \mu \mathrm{mol} / 1$ tanshinone IIA for $0,12,24$ and $48 \mathrm{~h}$, Apoptosis was assayed by annexin $\mathrm{V}$ and propidium iodide (PI) staining and analyzed by flow cytometry (FACScan, Becton Dickinson; Mountain View, CA) according to the manufacturer's protocol.

\section{Hoechst 33258 staining}

After the cells treated with $40 \mu \mathrm{mol} / 1$ tanshinone IIA for $0,12,24$ and $48 \mathrm{~h}$, the morphology of NB4 cells exposed to tanshinone IIA for different time was observed firstly under inverted microscope. effects of tanshinone IIA on cell adhesion, exponentially growing cells were incubated with tanshinone IIA $(40 \mu \mathrm{mol} / 1)$ for $24 \mathrm{~h}$ in a humidified $5 \% \mathrm{CO}_{2}$ atmosphere. The cells were centrifuged, washed twice with serum-free medium, counted, and resuspended in serum-free medium to a final concentration of $5 \times 10^{5}$ cells $/ \mathrm{ml} .1 \times 10^{5}$ cells were added to each well, and cells were allowed to adhere for $1 \mathrm{~h}$ at $37{ }^{\circ} \mathrm{C}$ in a humidified $5 \% \mathrm{CO}_{2}$ atmosphere. The nonadherent cells were removed by gently washing the cells with PBS, and then the adherent fraction was quantitated using MTT assays as described above.

\section{In vitro invasion assay}

The in vitro invasiveness of cells was assayed using a previously published method that uses Matrigelcoated Costar 24-well transwell cell culture chambers ("Boyden chambers") with 8.0-mm pore polycarbonate filter inserts. The chamber filters were coated with $50 \mathrm{mg} / \mathrm{ml}$ of Matrigel matrix, incubated overnight at room temperature under a 
laminar flow hood, and stored at $4{ }^{\circ} \mathrm{C}$. To study the effects of tanshinone IIA on the invasiveness of NB4 cells, exponentially growing cells were incubated with different concentrations of tanshinone IIA in $0.1 \%$ DMSO overnight. The cells were washed twice with serum-free RPMI 1640 containing $0.1 \%$ BSA, counted, and resuspended at $1 \times 10^{5}$ cells $/ \mathrm{ml}$. An $0.5-\mathrm{ml}$ cell suspension containing $1 \times 10^{5}$ cells in a serum-free RPMI 1640 containing tanshinone IIA or vehicle was added to the Matrigel-coated and rehydrated filter inserts. Next, $750 \mathrm{ml}$ of NIH fibroblast-conditioned medium was placed as a chemoattractant in 24-well plates, and the inserts were placed in wells and incubated at $37{ }^{\circ} \mathrm{C}$ for $24 \mathrm{~h}$. After the incubation period, the invasive cells that migrated into the lower chamber were counted under a light microscope. The invasive fractions of cells treated with tanshinone IIA were compared with those of DMSO $(0.1 \%)$-treated control cells, and the percentage inhibition of invasiveness was determined.

\section{Western blot analysis}

Cells were treated with $40 \mu \mathrm{mol} / 1$ tanshinone IIA for $0,12,24$ and $48 \mathrm{~h}$, then Western blotting analysis was used to detect the expression of Bcl-2 family members. Briefly, $2 \times 10^{5}$ cells were harvested and washed with ice-cold PBS twice and lysed for $30 \mathrm{~min}$ at $4{ }^{\circ} \mathrm{C}$, then debris was removed by centrifugation for $15 \mathrm{~min}$ at $15,000 \times g$ at $4{ }^{\circ} \mathrm{C}$, and equivalent amounts of protein were separated by $10 \%$ SDS-PAGE and transferred onto nitrocellulose filters. The filters were first stained to confirm uniform transfer of all samples and then incubated in blocking solution for $2 \mathrm{~h}$ at room temperature. The filters were reacted firstly with the primary antibodies at $4{ }^{\circ} \mathrm{C}$ overnight, followed by extensive washes with PBS twice and TBST twice. Filters were then incubated with horseradish peroxidase-conjugated secondary antibodies for $1 \mathrm{~h}$, washed with TBST and developed using the Super Signal West Pico Kit.

\section{Analysis of the mitochondrial membrane potential $(\Delta \psi m)$}

The mitochondrial membrane potential $(\Delta \psi \mathrm{m})$ were measured by FCM using the intramitochondrial dye JC-1(Alexis Biochemical Co., Germany) after NB4 cells treated with different concentrations of tanshinone IIA for $24 \mathrm{~h}$. The detection procedure was performed according to the manufacture's instructions. Data were converted to dot plots using Cell Quest software (Becton Dickison, Germany).

\section{Caspase activity assay}

After NB4 cells were treated $40 \mu \mathrm{mol} / \mathrm{l}$ tanshinone IIA for $0,12,24$ and $48 \mathrm{~h}$, the activity of caspases was determined by Caspase colorimetric assay kit, according to the manufacturer's protocol. Briefly, tanshinone IIA treated cells were washed with icecold PBS and lysed in a lysis buffer. The cell lysates were tested for protease activity using a caspasespecific peptide, conjugated to the color reporter molecule $p$-nitroanaline. The chromophore $p$-nitroanaline, cleaved by caspases, was quantitated with a spectrophotometer at a wavelength of $405 \mathrm{~nm}$. The caspase enzymatic activities in cell lysate are directly proportional to the color reaction.

Western blot analysis was also used to detect the variation of caspase- 3 after the cells exposed to $40 \mu \mathrm{mol} / 1$ tanshinone IIA for $24 \mathrm{~h}$. To confirm the contribution of caspase-3 to tanshinone IIA induced cell apoptosis, the cells were pretreated with a caspase-3-specific inhibitor, z-DEVD-FMK $(20 \mu \mathrm{mol} / \mathrm{l})$, prior to tanshinone IIA treatment. The cells were firstly treated with z-DEVD-FMK for $1 \mathrm{~h}$, then the cells were incubated with $40 \mu \mathrm{mol} / 1$ tanshinone IIA for $24 \mathrm{~h}$ and Western blot was used to detect the expression of caspase-3.

\section{Statistical analysis}

All experiments were performed in triplicate and the results were expressed as mean $\pm \mathrm{SD}$. Statistical analysis were performed with a Student's $t$-test using SAS 6.12 software. Statistical significance was accepted at the level of $p<0.05$.

\section{Results \\ Cell growth inhibition effects of tanshinone IIA on NB4 cells}

To investigate the cytotoxicity of tanshinone IIA on leukemia cells, NB4 cells were treated with 
various concentrations of tanshinone IIA for 12 , 24 and $48 \mathrm{~h}$. As shown in Figure 2, tanshinone IIA had significant growth inhibition effects on NB4 cells in a dose- and time-dependent manner. The inhibitory rate of tanshinone IIA between 30 and $50 \mu \mathrm{mol} / 1$ is much higher than that of lower concentrations of tanshinone IIA $(p<0.01)$. The IC50 values after 12,24 and $48 \mathrm{~h}$ of treatment were $45.8,24.7$ and $19.3 \mu \mathrm{mol} / 1$.

\section{Apoptosis assay}

To observe tanshinone IIA induced apoptosis, NB4 cells were treated with $40 \mu \mathrm{mol} / 1$ tanshinone IIA for $0,12,24$ and $48 \mathrm{~h}$, cells were collected and stained with annexin V and PI, and then subjected to flow cytometry assay to obtain an apoptosis scatter plot. As shown in Figure 3, by using double staining with annexin V and PI, apoptotic cells in the early-stage of apoptosis (positive for annexin $\mathrm{V}$ and negative for PI) is very higher, and the necrotic cells (both positive for annexin V and PI) is very lower, and the percentage of apoptotic cells in NB4 cells was increased in a time-dependent manner. Along with the enhancement of culture time, apoptotic cells gradually increased and the percentage of apoptotic cells reached up to over $60 \%$ when the cells were treated with $40 \mu \mathrm{mol} / 1$ tanshinone IIA for $48 \mathrm{~h}$ (Figure 3, M1).

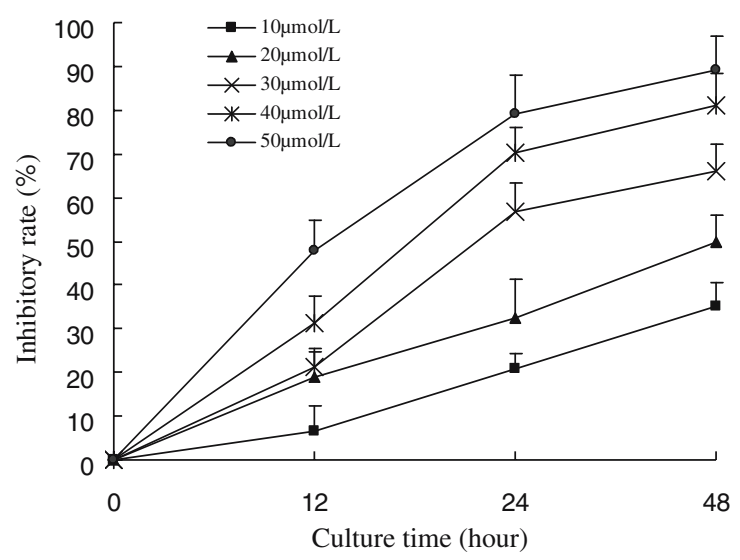

Figure 2. Cell growth inhibition caused by tanshinone IIA. After NB4 cells were treated with different concentrations of tanshinone IIA, MTT assay was used to detect cell growth inhibition as described in the 'Methods'. The inhibitory rate of tanshinone IIA between 30 and $50 \mu \mathrm{mol} / 1$ is much higher than that of lower concentrations of tanshinone IIA $(p<0.01)$.

\section{Hoechst 33258 staining}

After cells treated with $40 \mu \mathrm{mol} / 1$ tanshinone IIA for $0,12,24$ and $48 \mathrm{~h}$, marked morphological changes of cell apoptosis such as condensation of chromatin and nuclear fragmentations were found clearly using Hoechst 33258 staining (Figure 4). Apoptotic cells gradually increased in time-dependent manner in NB4 cells.

\section{Expression of apoptosis related genes}

To clarify the mechanism of tanshinone IIA induced apoptosis in NB4 cells, the expressions of Bcl-2 family members were detected after the cells treated with $40 \mu \mathrm{mol} / 1$ tanshinone IIA for 0 , 12, 24 and $48 \mathrm{~h}$. The results revealed that the expression of bcl-2 and bcl-xl was down-regulated remarkably while bax expression was up-regulated concurrently after the cells were treated with tanshinone IIA, and the expressions of other Bcl2 family members including bid, bak and bad remained constant before and after apoptosis occurred (Figure 5).

\section{Tanshinone IIA inhibit NB4 cell adhesion and invasion}

The ECM proteins to which tumor cells initially attach include laminin, fibronectin, type IV collagen, and vitronectin. Laminin, fibronectin, vitronectin, and collagen have been found in the basal lamina that promote the adhesion and invasion of tumor cells. To determine whether tanshinone IIA affects the integrin-mediated cell adhesion to ECM, NB4 cells were incubated with tanshinone IIA for $24 \mathrm{~h}$, and then the integrin-mediated cell adhesion was examined. As shown in Figure 6, pretreatment of NB4 cells with tanshinone IIA inhibited their adhesion to laminin-, fibronectin-, collagen- and vitronectin-coated plates. Matrigel matrix-coated Boyden chambers were used to examine the ability of tanshinone IIA to inhibit the invasiveness of NB4 cells. The cells were treated with different concentrations of tanshinone IIA overnight and then placed in Matrigel matrixcoated Boyden chambers and allowed to invade for $24 \mathrm{~h}$. We observed that pretreatment with different concentrations of tanshinone IIA inhibited the invasiveness of NB4 cells in a dosedependent manner (Figure 7). 

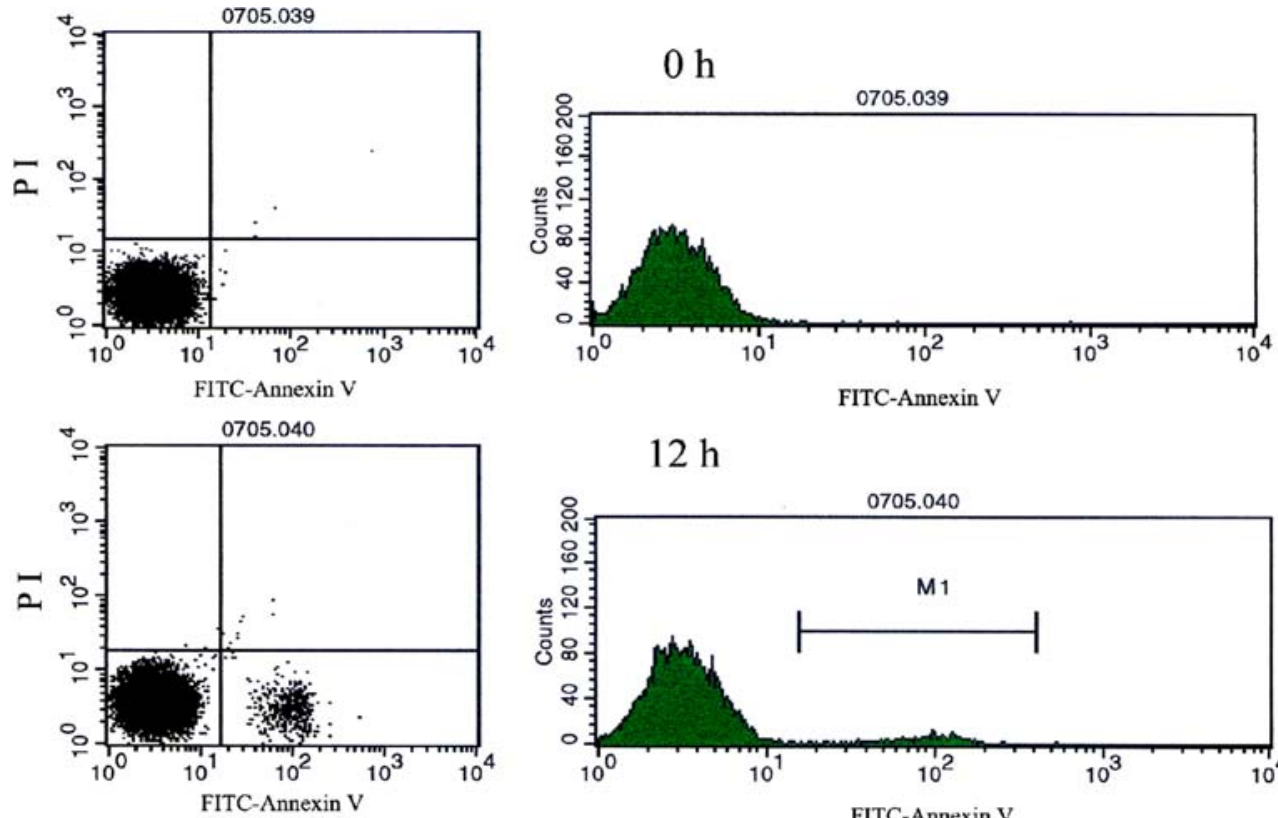

$12 \mathrm{~h}$
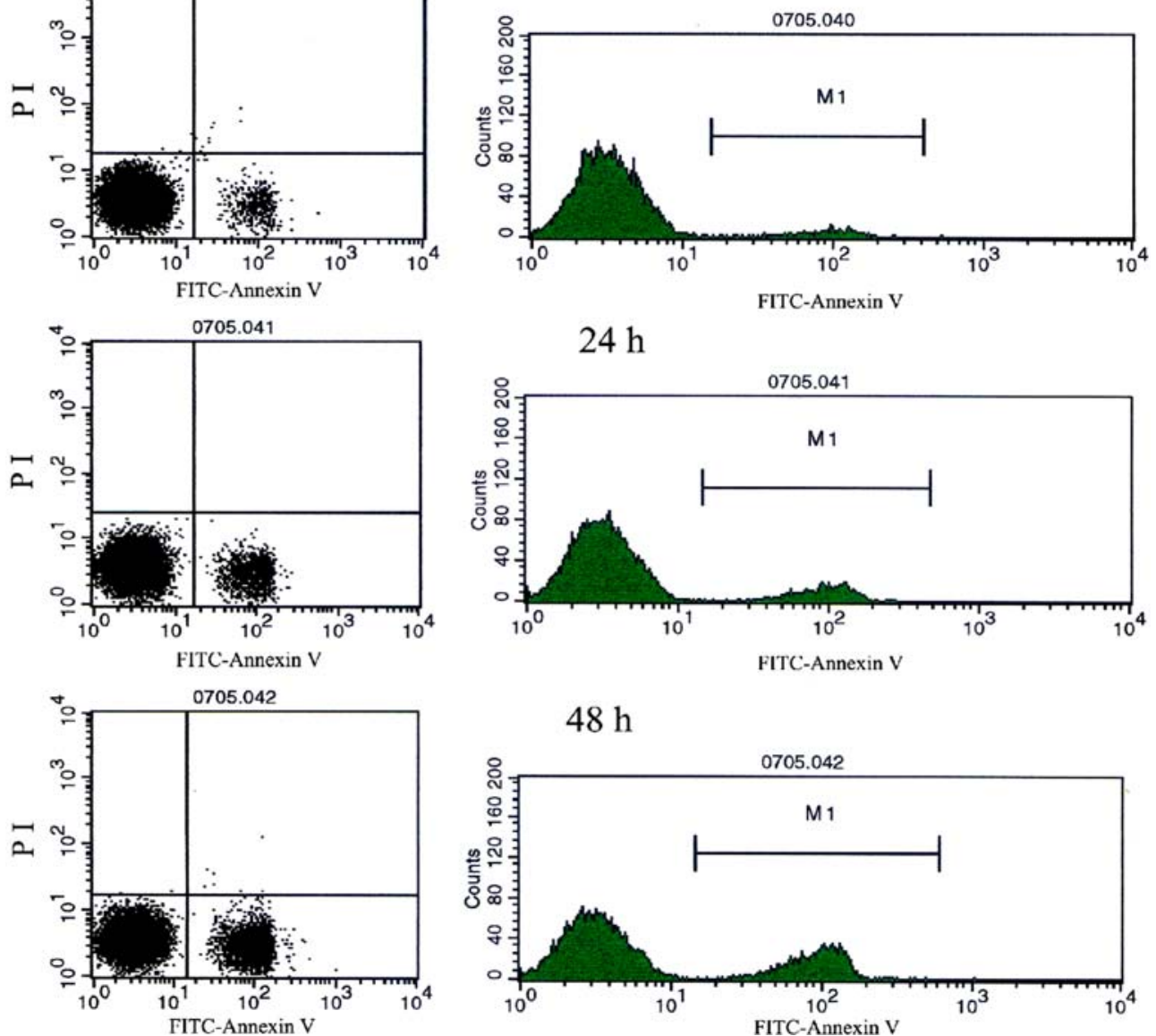

Figure 3. Cell apoptosis caused by tanshinone IIA. After NB4 cells were treated with $40 \mu \mathrm{mol} / 1$ tanshinone IIA for $0,12,24$ and $48 \mathrm{~h}$, cell apoptosis was analyzed by flow cytometry (double staining with annexin V and PI) as described in the 'Methods'. Tanshinone IIA could induce apoptosis when cultured with NB4 cells for 12-48 h, the apoptotic cells (positive for annexin V and negative for PI) were very higher, and the cell aoptotic rate (M1) gradually increased in a time-dependent manner.

\section{Disruption of the $\Delta \psi m$}

The changes in the membrane potential of the mitochondria in tanshinone IIA treated cells were examined after the cells were treated for $24 \mathrm{~h}$. The results showed that NB4 cells lost their mitochon- drial membrane potential following tanshinone IIA treatment. After tanshinone IIA treatment for $24 \mathrm{~h}$, the cells exhibited a significant alterations in $\Delta \psi \mathrm{m}$, and the percentage of disruption of $\Delta \psi \mathrm{m}$ gradually increased in a dose-dependent manner (Figure 8). This suggests that tanshinone IIA 

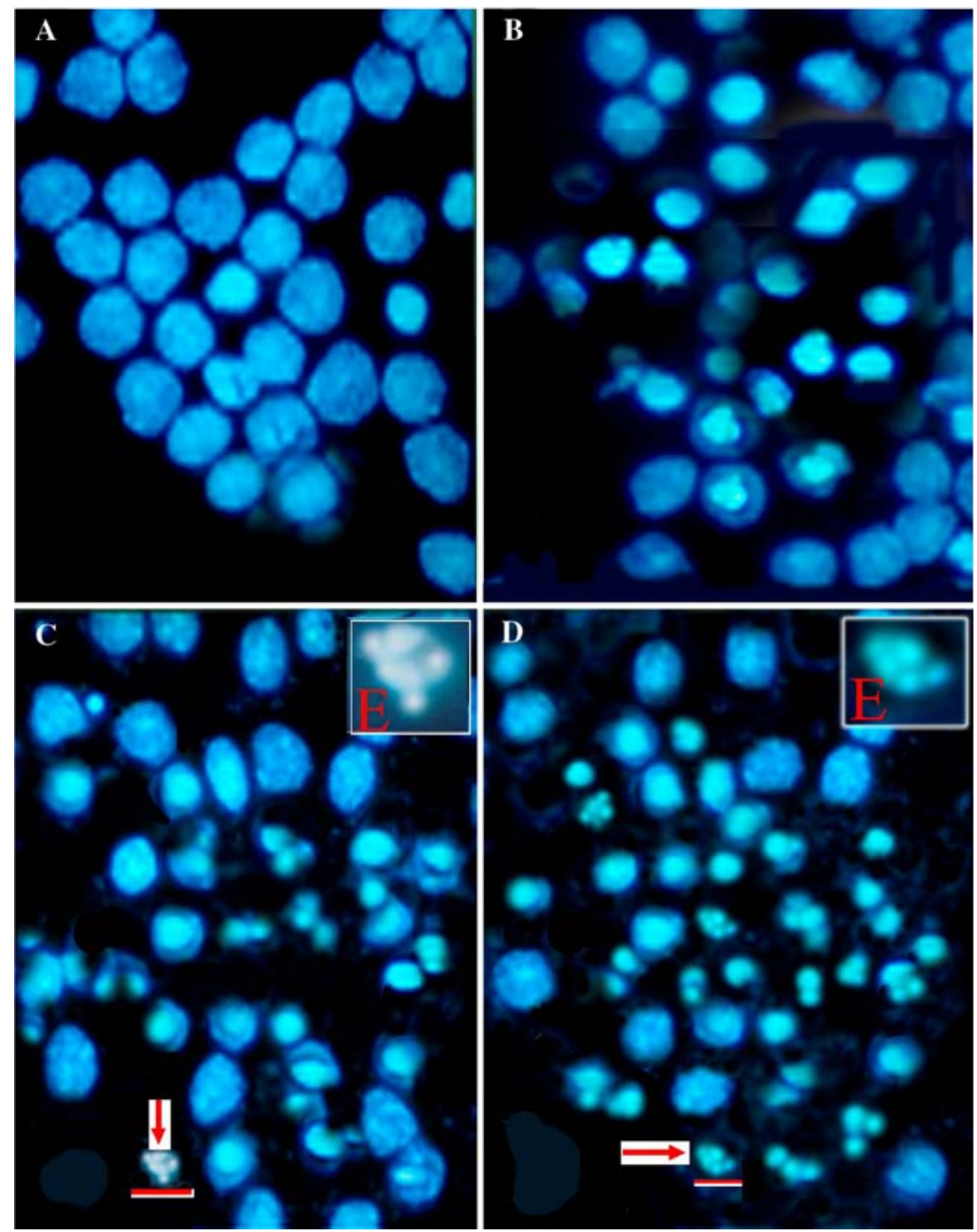

Figure 4. Cell apoptosis observed by Hoechst 33258 staining $(200 \times$ magnification). After NB4 cells exposed to $40 \mu \mathrm{mol} / 1$ tanshinone IIA for $0,12,24$ and $48 \mathrm{~h}$, Hoechst 33258 staining was used to observe the apoptotic cells as described in the 'Methods'. Apoptotic cells gradually increased in a time-dependent manner, and marked morphological changes of cell apoptosis including condensation of chromatin and nuclear fragmentation were found clearly after the cells treated for different times. (A) $0 \mathrm{~h}$; (B) $12 \mathrm{~h}$; (C) $24 \mathrm{~h}$; (D) $48 \mathrm{~h}$; (E) $400 \times$ magnification. Arrow: the apoptotic cell displaying apoptotic bodies and nuclear fragmentation. $\mathrm{Bar}=20 \mu \mathrm{m}$.

induced apoptosis involves mitochondrial signaling pathway.

\section{Caspase-3 activities in tanshinone IIA-induced apoptosis}

To understand the activation of caspase cascade during tanshinone IIA induced apoptosis in leukemia cells, we first investigated various caspase specific activities after the cells were treated with $40 \mu \mathrm{mol} / 1$ tanshinone IIA for $0,12,24$ and $48 \mathrm{~h}$.
As shown in Figure 9, the colorimetric assay of caspase activities revealed that caspase- 3 activities were increased remarkably in a dose-dependent manner.

After the cells exposed to $40 \mu \mathrm{mol} / 1$ tanshinone IIA for $24 \mathrm{~h}$, Western blot analysis was also used to detect the variation of caspase-3. As shown in Figure 10A, Caspase-3 was activated by the loss of caspase- 3 proenzyme $(32-\mathrm{kD})$ and the appearance of its $20-\mathrm{kD}$ subunit after the cells were incubated over $24 \mathrm{~h}$. 


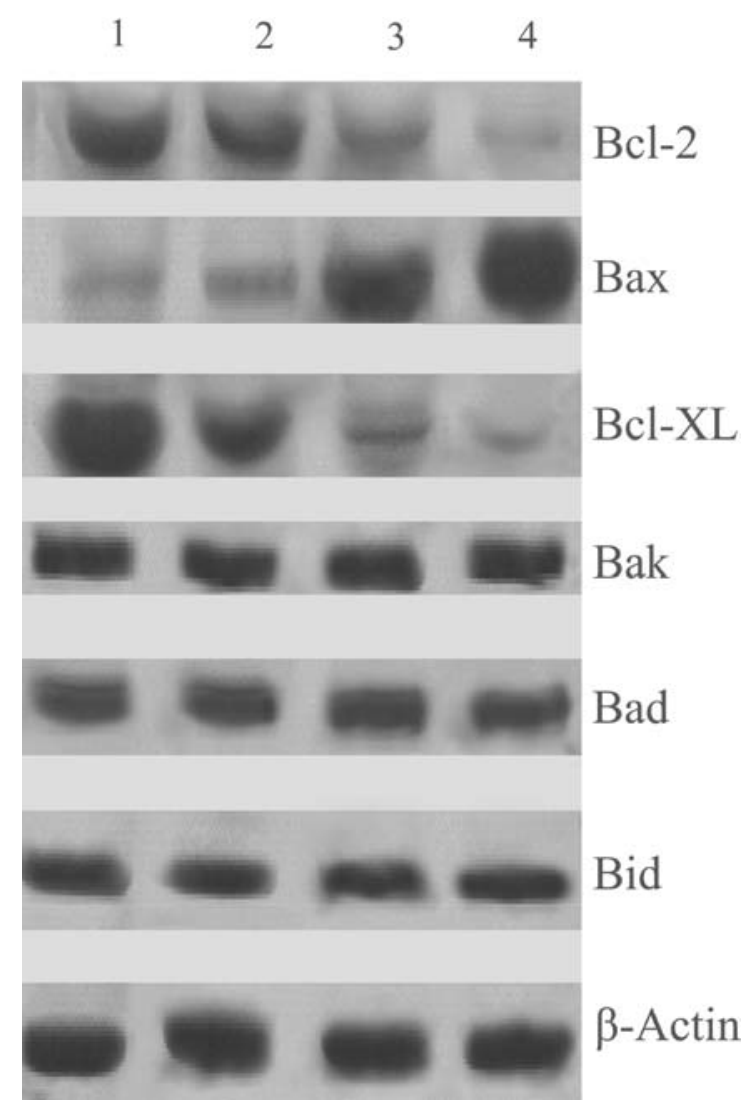

Figure 5. Western blot analysis of apoptosis related genes. After NB4 cells treated with $40 \mu \mathrm{mol} / 1$ tanshinone IIA for 0 , 12,24 and $48 \mathrm{~h}$, Western blot analysis was used to detect the variation of Bcl-2 family as described in the 'Methods'. The expressions of bcl-2 and bcl-xl were down-regulated while Bax expression was up-regulated, and the expressions of other Bcl2 family members including bid, bak and bad remained constant before and after apoptosis occurred. Lane 1,2, 3 and 4 were: $0,12,24$ and $48 \mathrm{~h}$. $\beta$-actin as an internal control.

To estimate the contribution of caspase- 3 to tanshinone IIA induced cell apoptosis, the cells were pretreated with a caspase-3-specific inhibitor. As shown in Figure 10B, $1 \mathrm{~h}$ pretreatment of NB4 cells with z-DEVD-FMK could block tanshinone IIA induced activation of caspase-3, indicating that tanshinone IIA caused apoptosis was mediated via the activation of caspase-3.

\section{Discussion}

Herbal medicines have always held an attraction for patients. Approximately $25 \%$ of prescription medicines are derived from plants. It is currently estimated that over $50 \%$ of all patients diagnosed with cancer explore complementary and alternative medicine especially herbal medicine. In recent years, the use of herbal medicines to prevent the development or recurrence of cancers has become widely accepted as a realistic option for the treatment of malignant disease and herbal medicines have been proved to play an important role in integrative cancer treatment $[4,11]$. Tanshinone IIA (as shown in Figure 1), a derivative of phenanthrene-quinone isolated from Danshen, is now widely used in the treatment of inflammatory and cardiological disease. Previous studies have shown that tanshinone IIA has a large variety of pharmacological activities such as inhibition of clotting [12], inhibition of NO synthase [13] and dosedependent inhibition on the basic fibroblast growth factor (bFGF)-induced human Smooth muscle cell (SMC) proliferation [14]. Recent data have demonstrated that tanshinone IIA has anti-cancer activities on a large variety of cancer cells including solid tumor $[9,10]$ as well as lymphocytic leukemia cells [15].

In this study, we found that tanshinone IIA could inhibit cell growth, induce apoptosis by activation of caspase- 3 and disruption of the mitochondrial membrane potential, as well as reduce cell adhesion to and invasion through ECM on leukemia NB4 cells in vitro. Western blotting analysis demonstrated that antiapoptotic protein bcl-2 was downregulatd while pro-apoptotic protein bcl-xl upregulated remarkably in a time-dependent manner when apoptosis occurred. We therefore conclude that tanshinone IIA has significant anti-proliferation effect by induction of apoptosis via activation of caspase- 3 and disruption of mitochondrial membrane potential, as well as by downregulation of anti-apoptotic protein bcl-2 and bcl-xl and upregulation of proapoptotic protein bax. Furthermore, tanshinone IIA demonstrate significant inhibition effects on cell adhesive and invasive ability in leukemia NB4 cells in vitro.

The dissemination of cancer occurs via shedding of cells from the primary tumor with subsequent seeding, followed by invasion and proliferation at the secondary site [16]. The extracellular matrix (ECM) serves as a scaffold to promote the cellular responses of adhesion, proliferation, and migration during tumor cell growth [17]. ECM is a prerequisite for the structural and functional homeostasis of bone marrow (BM) 


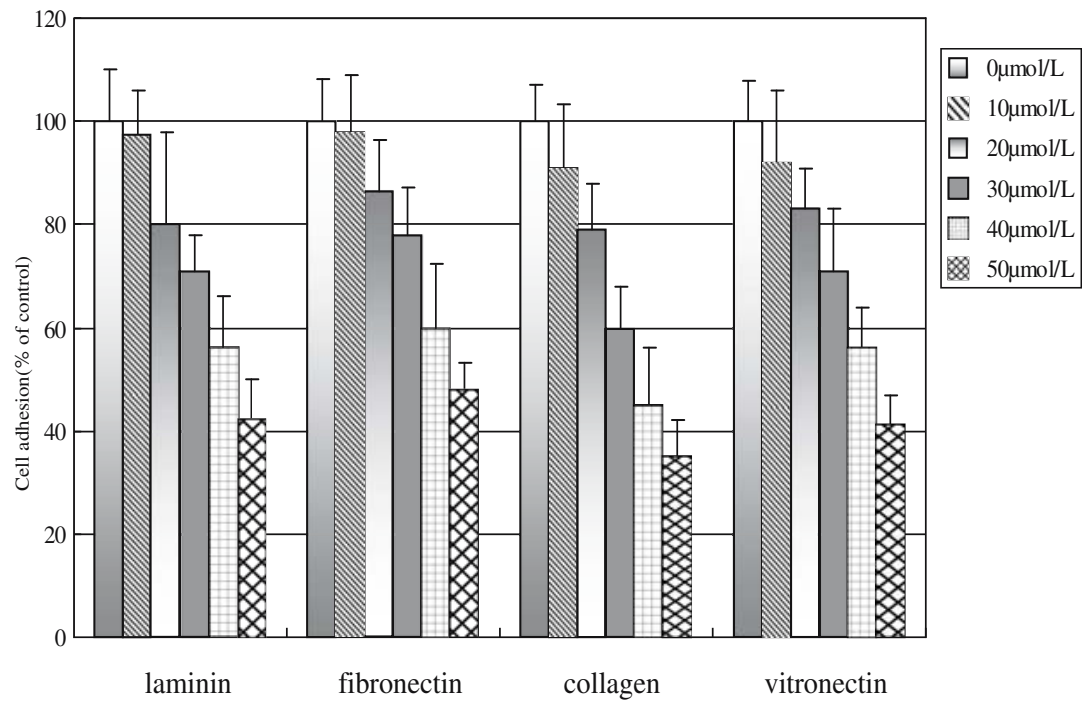

Figure 6. Tanshinone IIA inhibited adhesive property of NB4 cells. NB4 cells were incubated with different concentrations of tanshinone IIA for $24 \mathrm{~h}$, and then processed for adhesion assays using laminin-, fibronectin-, collagen type IV, or vitronectin-coated 96-well plates as described in "Materials and Methods". The results showed that cell adhesive cells were gradually reduced in a dose-dependent manner.

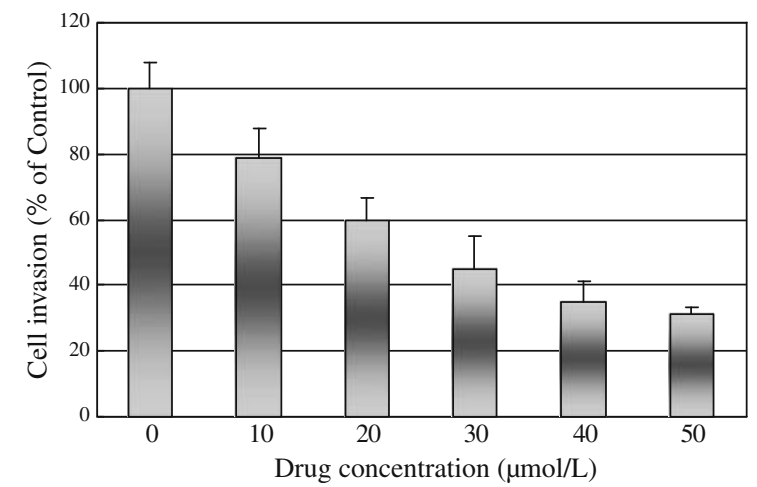

Figure 7. Tanshinone IIA inhibited invasive property of NB4 cells. NB4 cells were treated with different concentrations of tanshinone IIA overnight and then placed in Matrigel matrixcoated Boyden chambers and allowed to invade for $24 \mathrm{~h}$ as described in "Materials and Methods". The results showed that tanshinone IIA inhibited the invasiveness of NB4 cells in a dose-dependent manner, the amount of invasive cells gradually decreased after treatment with different concentrations of tanshinone IIA.

microenvironment. The role of ECM in physiologic hematopoiesis and its pathologic change are very important in the development of leukemia, and cell-ECM interactions may trigger leukemia cell proliferation, survival and dissemination [18]. Therefore, adhesion to and invasion through ECM contributes to enlarged leukemia cell dissemination, proliferation, and may cause the poor clinical

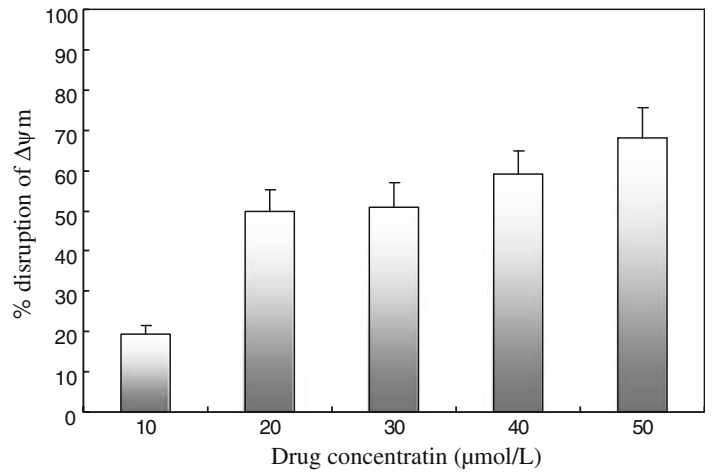

Figure 8. Disruption of the $\Delta \psi \mathrm{m}$. The changes in the membrane potential of the mitochondria in tanshinone IIA treated cells were detected as described in "Materials and Methods". After tanshinone IIA treatment for $24 \mathrm{~h}$, the NB4 cells exhibited a significant alterations in $\Delta \psi \mathrm{m}$, and the percentage of disruption of $\Delta \psi \mathrm{m}$ gradually increased in a dose-dependent manner.

outcome in leukemia patients. In this study, our results demonstrated that tanshinone IIA could inhibit the effects on cell adhesive and invasive ability in leukemia NB4 cells in vitro, indicating tanshinone IIA may serve as a potential reagent in the treatment of APL.

The caspases are a family of intracellular cysteine proteases with specificity for aspartic acid residues [19]. Two of these groups, named "initiator" and "effector" caspases, play an important 


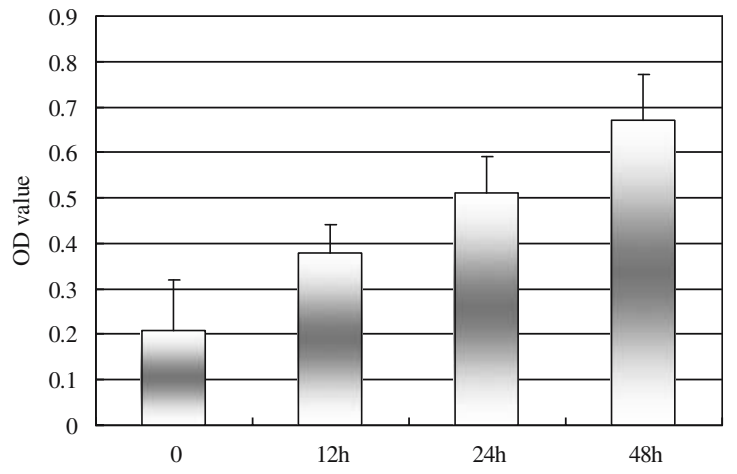

Figure 9. The variation of caspase- 3 activities caused by tanshinone IIA. After NB4 cells were treated with $40 \mu \mathrm{mol} / 1$ tanshinone IIA for $0,12,24$ and $48 \mathrm{~h}$, caspase- 3 activities were detected as described in "Materials and Methods". The results revealed that caspase-3 activities were increased remarkably in a time-dependent manner.

roles in the apoptotic process [20]. Caspases-3 is one of the most important executioner, which is capable of cleaving many important cellular substrates, and caspase- 3 mediated cell death plays an important role in pathogenesis and therapy of a variety of hematological malignancies [21]. Our results demonstrated that tanshinone IIA induced apoptosis was related to activation of caspase-3, and caspase-3 specific inhibitor z-DEVD-FMK could block tanshinone IIA induced activation of caspase-3, indicating that tanshinone IIA caused apoptosis in NB4 cells was mediated via the activation of caspase-3.

The Bcl-2 family consists of about 20 homologs of important apoptotic regulators of programmed cell death. This family of proteins now includes both anti-apoptotic molecules such as Bcl-2 and Bcl-XL, and pro-apoptotic molecules such as Bax, Bak, Bid and Bad [22]. Cancers with high levels of Bcl-2 and $\mathrm{Bcl}-\mathrm{XL}$ proteins are resistant to drug induced apoptosis in a wide spectrum of chemotherapeutic agents, so Bcl-2 as well as Bcl-XL have become attractive targets for designing new anti-cancer drugs [23]. Pro-apoptotic members of the Bcl-2 family, especially Bax and Bid, play important roles in drug induced apoptosis by control of mitochondrial permeability due to their ability to form channels in membranes and to regulate preexisting channels [24]. In this study, our results revealed that treatment by tanshinone IIA in NB4 cells caused upregulation of bax as well as downregulation of bcl-2 and bcl-xl followed by disruption of mitochondrial membrane potential. This inidicates that

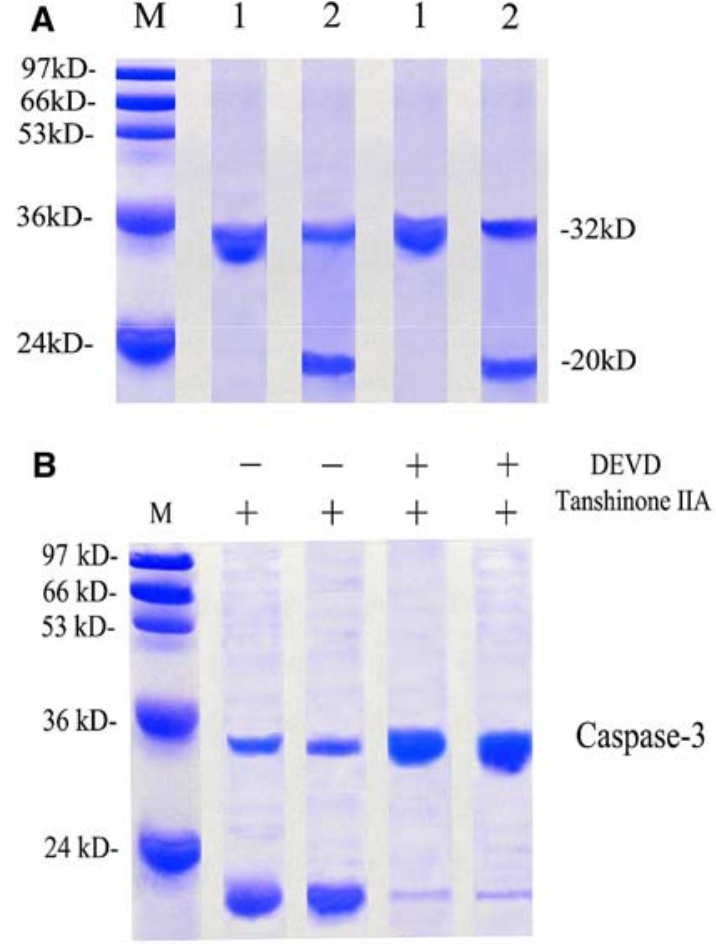

Figure 10. Western blot analysis of caspase-3. (A) Caspase-3 was activated by the loss of caspase- 3 proenzyme $(32-\mathrm{kD})$ and the appearance of its $20-\mathrm{kD}$ subunit after the cells exposed to $40 \mu \mathrm{mol} / \mathrm{l}$ tanshinone IIA for $24 \mathrm{~h}$. M, Molecular weight marker; lane 1 , cells treated with $0 \mu \mathrm{mol} / 1$ tanshinone IIA; lane 2 , cells treated with $40 \mu \mathrm{mol} / 1$ tanshinone IIA. (B) Tanshinone IIA caused activation of caspase- 3 was blocked by caspase- 3 specific inhibitor. The cells were firstly treated with z-DEVD-FMK for $1 \mathrm{~h}$, then the cells were incubated with $40 \mu \mathrm{mol} / 1$ tanshinone IIA for $24 \mathrm{~h}$. The results showed that pretreatment of NB4 cells with z-DEVD-FMK could block tanshinone IIA induced activation of caspase-3, indicating that tanshinone IIA caused apoptosis was mediated via the activation of caspase-3.

mitochondrial signaling pathway was involved in tanshinone IIA induced apoptosis in APL cells.

In summary, our data provide a potential mechanism for tanshinone IIA-induced apoptosis and cell growth inhibition in leukemia NB4 cells, suggesting that tanshinone IIA may serve as an effective adjunctive reagent for the treatment of leukemia, and that in vivo anti-cancer effects as well as its potential clinical effectiveness need further investigation.

\section{Acknowledgements}

We thank post-doctoral fellow Liu DJ for help with computer analysis and presentation of data. 
We also thank the members of our laboratories for their insight and technical support. This work is supported by the grants from National Natural Foundation of China (No. 30570786, No. 30472022).

\section{References}

1. Jabbour E.J., Estey E. and Kantarjian H.M., Adult acute myeloid leukemia. Mayo. Clin. Proc. 81: 247-260, 2006.

2. Macheta M. and Yin J.A., Recent advances in the treatment of AML. Hematol. Oncol. 19: 107-118, 2001.

3. Lengfelder E., Saussele S., Weisser A., Buchner T. and Hehlmann R., Treatment concepts of acute promyelocytic leukemia. Crit. Rev. Oncol. Hematol. 56: 261-274, 2005.

4. Boon H. and Wong J., Botanical medicine and cancer: a review of the safety and efficacy. Expert. Opin. Pharmacother. 5: 2485-2501, 2004.

5. Zhou L., Zuo Z. and Chow M.S., Danshen: an overview of its chemistry, pharmacology, pharmacokinetics, and clinical use. J. Clin. Pharmacol. 45: 1345-1359, 2005.

6. Wang A.M., Sha S.H., Lesniak W. and Schacht J., Tanshinone (Salviae miltiorrhizae extract) preparations attenuate aminoglycoside-induced free radical formation in vitro and ototoxicity in vivo. Antimicrob. Agents Chemother. 47: 1836-1841, 2003.

7. Yang L.J., Jeng C.J., Kung H.N., Chang C.C., Wang A.G., Chau G.Y., Don M.J. and Chau Y.P., Tanshinone IIA isolated from Salvia miltiorrhiza elicits the cell death of human endothelial cells. J. Biomed. Sci. 12: 347-361, 2005.

8. Choi H.S., Cho D.I., Choi H.K., Im S.Y., Ryu S.Y. and Kim K.M., Molecular mechanisms of inhibitory activities of tanshinones on lipopolysaccharide-induced nitric oxide generation in RAW 264.7 cells. Arch. Pharm. Res. 27: 1233-1237, 2004.

9. Wang X., Wei Y., Yuan S., Liu G., Lu Y., Zhang J. and Wang W., Potential anticancer activity of tanshinone IIA against human breast cancer. Int. J. Cancer. 116: 799-807, 2005.

10. Yuan S.L., Wei Y.Q., Wang X.J., Xiao F., Li S.F. and Zhang J., Growth inhibition and apoptosis induction of tanshinone II-A on human hepatocellular carcinoma cells. World J. Gastroenterol. 10: 2024-2028, 2004.
11. Treasure J., Herbal medicine and cancer: an introductory overview. Semin. Oncol. Nurs. 21: 177-183, 2005.

12. Chan T.Y., Interaction between warfarin and danshen (Salvia miltiorrhiza). Ann. Pharmacol. 35: 501-504, 2001.

13. Yokozawa T. and Chen C.P., Role of Salvia miltiorrhiza radix extract and its compounds in enhancing nitric oxide expression. Phytomedicine 7: 55-61, 2000.

14. Zhou G.Y., Zhao B.L., Hou J.W., Ma G.E. and Xin W.J., Protective effects of sodium tanshinone IIA sulphonate against adriamycin-induced lipid peroxidation in mice hearts in vivo and in vitro. Pharmacol. Res. 40: 487-491, 1999.

15. Mosaddik M.A., In vitro cytotoxicity of tanshinones isolated from Salvia miltiorrhiza Bunge against P388 lymphocytic leukemia cells. Phytomedicine 10: 682-685, 2003.

16. Vasiliev J.M., Cytoskeletal mechanisms responsible for invasive migration of neoplastic cells. Int. J. Dev. Biol. 48: 425-439, 2004

17. Simpson-Haidaris P.J. and Rybarczyk B., Tumors and fibrinogen. The role of fibrinogen as an extracellular matrix protein. Ann. N.Y. Acad. Sci. 936: 406-425, 2001.

18. Yu X.F. and Han Z.C., Matrix metalloproteinases in bone marrow: roles of gelatinases in physiological hematopoiesis and hematopoietic malignancies. Histol. Histopathol. 21: 519-531, 2006.

19. Denault J.B. and Salvesen G.S., Caspases: keys in the ignition of cell death. Chem. Rev. 102: 4489-4500, 2002.

20. Philchenkov A.A., Caspases as regulators of apoptosis and other cell functions. Biochemistry 68: 365-376, 2003.

21. Smolewski P., Darzynkiewicz Z. and Robak T., Caspasemediated cell death in hematological malignancies: theoretical considerations, methods of assessment, and clinical implications. Leuk. Lymphoma 44: 1089-1104, 2003.

22. Kirkin V., Joos S. and Zornig M., The role of Bcl-2 family members in tumorigenesis. Biochim. Biophys. Acta 1644: 229-249, 2004

23. Wang S., Yang D. and Lippman M.E., Targeting Bcl-2 and Bcl-XL with nonpeptidic small-molecule antagonists. Semin. Oncol. 30(S16) 133-142, 2003.

24. Sharpe J.C., Arnoult D. and Youle R.J., Control of mitochondrial permeability by Bcl-2 family members. Biochim. Biophys. Acta 1644: 107-113, 2004. 